1 Conselho Nacional de Desenvolvimento Científico e Tecnológico (CNPq) - Brasília (DF), Brasil.

gilberto.souza@cnpq.br

2 Universidade Federal do Rio Grande do Sul (UFRGS), Programa de Pós-Graduação em Educação em Ciências Porto Alegre (RS), Brasil. luciana.calabro@ufrgs.br

\section{Avaliação do grau de implantação do Programa Pesquisa para o SUS: gestão compartilhada em saúde}

\author{
Evaluation of the degree of implementation of the Program Research \\ for SUS: shared management in health
}

Gilberto Ferreira de Souza', Luciana Calabró

RESUMO O estudo objetivou monitorar a execução e o grau de implantação do Programa de Pesquisa para o SUS: gestão compartilhada em saúde, no País. Utilizou como estratégia de pesquisa o estudo de casos múltiplos, na busca ativa nos documentos do programa e no Sistema Pesquisa Saúde, do Ministério da Saúde. O programa lançou cinco edições e investiu R 274,5 milhões na contratação de 2.923 projetos de pesquisa, tendo sido executado de forma descentralizada. Está 'implantado’ em 20 estados, e ‘parcialmente implantado’ nos demais. O êxito da ação pode ser medido pelo aporte de recursos federais e estaduais, pela adesão da comunidade científica e dos gestores do Sistema Único de Saúde (SUS).

PALAVRAS-CHAVE Monitoramento. Avaliação. Política de pesquisa em saúde. Prioridades em pesquisa. Financiamento.

\begin{abstract}
The study aimed to monitor the accomplishment and the degree of implementation of the Program 'Research for SUS: shared management in health', in Brazil. A multiple case design strategy was employed, involving an active search in the Programs' documents and in the Health Research System of the Ministry of Health. The Program launched 5 editions, invested $R \$$ 274.5 million in the hiring of 2.923 research projects, and had a decentralized implementation. The results indicate that the Project was considered 'Implemented' in 20 states, and 'Partially implemented' in the others. The success of the intervention can be due to the contribution of federal and state resources, the adherence of the scientific community and of the Brazilian Unified Health System (SUS) managers.
\end{abstract}

KEYWORDS Monitoring. Evaluation. Health Research Policy. Priorities in research. Financing. 


\section{Introdução}

No Brasil, país com dimensões continentais, há desigualdades regionais e intrarregionais nos campos socioeconômico e científico (BARROS, 2000) que necessitam ser enfrentadas. Gerar políticas públicas que promovam a desconcentração dos investimentos direcionados ao desenvolvimento científico e tecnológico, contemplando essas diferenças, apresenta-se como um dos grandes desafios nacionais.

No campo da saúde, utilizar a descentralização de recursos e, também, do poder decisório como modelo é condição sine qua non para o sucesso e a efetividade das políticas públicas. A descentralização do fomento em Ciência, Tecnologia e Inovação (CTI) no País é recente, e vem contribuindo de forma significativa para o fortalecimento do Sistema Nacional de Ciência, Tecnologia e Inovação (SNCTI) e a inserção de novos atores institucionais (CGEE, 2010).

$\mathrm{Na}$ vanguarda das ações no campo da saúde, o Ministério da Saúde (MS), por intermédio do Departamento de Ciência e Tecnologia (Decit), deu início a um processo de institucionalização da Ciência, Tecnologia e Inovação em Saúde (CTIS) no País, com a criação da Política Nacional de Ciência e Tecnologia em Saúde (PNCTS) e a Agenda Nacional de Prioridades de Pesquisa em Saúde (ANPPS) (BRASIL, 2011B).

Esses marcos institucionais foram fundamentais para posicionar o MS como indutor, financiador e usuário do CTIS no Brasil. Dentre as inovações, já em 2002, o MS, via Decit, lançou o modelo descentralizado de política pública no contexto do fomento à pesquisa, no setor saúde, denominado Projeto Gestão Compartilhada, do qual participaram dez unidades federativas (UF): Alagoas, Bahia, Ceará, Minas Gerais, Mato Grosso do Sul, Paraíba, Pernambuco, Piauí, Sergipe e Santa Catarina (BRASIL, 2011A).

O projeto foi formatado com o ambicioso propósito de financiar pesquisas que pudessem contribuir para a resolução dos problemas prioritários de saúde e para o fortalecimento da gestão do Sistema Único de Saúde (SUS).
Em 2003, o MS firmou acordo de cooperação com o Ministério da Ciência e Tecnologia e Inovação (MCTI). Essa parceria possibilitou a celebração de termo de cooperação com o Conselho Nacional de Desenvolvimento Científico e Tecnológico (CNPq), que passou a ser o gestor administrativo do projeto. Em 2004, o projeto foi renomeado como Programa Pesquisa para o SUS: gestão compartilhada em saúde (PPSUS), com o objetivo de aperfeiçoar a gestão, assim como os serviços ofertados pelo SUS à população (BRASIL, 2014B).

A execução de todas as etapas do programa baseia-se no documento 'Diretrizes Técnicas' (DDT) do PPSUS, que estabelece o rito, desde a definição de prioridades de pesquisa até $\mathrm{o}$ sistema de acompanhamento e avaliação (A\&A) dos projetos aprovados. Este documento vem sendo remodelado desde a primeira edição do programa, tendo sua última atualização ocorrido em 2014 (BRASIL, 2014B).

Quatro instituições participam da gestão do programa: no âmbito federal, o MS e o CNPq; e na esfera estadual, as Fundações de Amparo/ Apoio à Pesquisa (FAP) e as Secretarias Estaduais de Saúde (SES). O MS é o coordenador nacional do programa, subsidiado pela expertise do CNPq na formatação de editais, nos processos de seleção de projetos e atividades de A\&A das pesquisas aprovadas. As FAP e as SES são responsáveis, conjuntamente, pelas oficinas de definição de prioridades (BRASIL, 2009), pela contratação dos projetos aprovados e pelas atividades de A\&A. Os papéis dessas instituições são complementares e compartilhados, conforme a atividade que deve ser operacionalizada em cada instante, sem hierarquizar o processo decisório.

O programa prevê o lançamento de edições a cada dois anos. A primeira ocorreu em 2002, tendo continuidade nos anos de 2004, 2006, 2008 e 2012. Em 2010, o Decit, da Secretaria de Ciência, Tecnologia e Insumos Estratégicos (SCTIE), do MS, consultando o CNPq, decidiu cancelar o lançamento da respectiva edição. Essa decisão foi tomada baseada 
na necessidade que algumas FAP demonstraram de prorrogar a vigência dos projetos de pesquisa ainda em execução, contratados nas edições de 2006 e 2008.

Em 2011, foi lançada uma edição especial do programa, incorporando ao seu objeto a 'implantação das redes de atenção à saúde nos estados'. Participaram dessa edição as seguintes UF: Amazonas, Ceará, Minas Gerais, Mato Grosso do Sul, Pará, Pernambuco, Paraná, São Paulo, Santa Catarina e Distrito Federal. Na perspectiva do MS, a implantação dessas redes contribuiria para aprimorar a organização do SUS (BRASIL, 2014A).

Embora o programa preveja o lançamento de edições bianuais, sua implementação fica condicionada à abertura de editais e/ou chamadas públicas pelas FAP e à efetiva contratação dos projetos aprovados. Em algumas UF, há a necessidade de lançamento de novos editais, por exemplo, para contemplar linhas de pesquisa previstas no edital inicial e que não obtiveram propostas aprovadas.

Considerando a magnitude desse programa, além dos investimentos - que totalizaram aproximadamente $\mathrm{R} \$ 274,5$ milhões, na contratação de 2.923 projetos de pesquisa -, e considerando não ter sido identificada, na literatura especializada, a realização de avaliação dessa intervenção, este estudo objetivou mapear a execução do PPSUS e avaliar seu grau de implantação nas UF, no período de 2004 a 2012.

Em virtude da polissemia conceitual acerca do tema avaliação, adotou-se, neste trabalho, a definição proposta por Ligia Maria Vieira-daSilva no livro 'Avaliação de políticas e programas de saúde', que a concebe como

um julgamento que se faz sobre uma intervenção sanitária (política, programa ou prática), voltada para a resolução de problemas de saúde, visando aferir o mérito, esforço ou valor da referida intervenção ou do seu produto, para o seu aperfeiçoamento. (VIEIRA-DA-SILVA, 2014, P. 16).

A autora afirma, também, que a avaliação "é um dos componentes do processo de gestão”.
Chen (1990) propõe que a avaliação seja focada na 'teoria do programa', o que permite visualizar os fatores e os processos que geram os efeitos observados em uma dada intervenção. Segundo o autor, essa abordagem facilita o processo de coleta e a análise de dados, partindo do modus operandi do programa, que é demonstrado através de seu modelo lógico.

Oliveira (2008), em conjunto com os stakeholders do PPSUS, construiu o Modelo Lógico(ML) e a Matriz de Medidas Avaliativas (MMA) do programa, que explicitam sua teoria. O modelo foi constituído a partir da tríade 'componentes, elementos e atividades'. Seus componentes são a gestão compartilhada, a aproximação entre os sistemas de saúde e de ciência e tecnologia estaduais; financiamento e sustentabilidade do programa, as prioridades de pesquisa e a seleção de projetos; acompanhamento e avaliação das pesquisas, e o desenvolvimento da capacidade regional de pesquisa. Seus elementos foram constituídos dos objetivos, na implantação; dos processos, dos efeitos e do resultado final. Segundo a autora, esses componentes e elementos, com suas respectivas atividades, representam a teoria do PPSUS expressa no ML.

Entre as características ou atributos da avaliação formatados por Vieira-da-Silva \& Formigli (1994), e revisadas por Vieira-daSilva (2005), o grau de implantação da intervenção configura-se como uma alternativa de avaliação do processo de implantação de programas de governo.

\section{Metodologia}

$\mathrm{O}$ estudo dividiu-se em duas partes. $\mathrm{Na}$ primeira, destinada a mapear as UF participantes por edição do programa, e a quantificar os recursos investidos, o número de projetos aprovados e as subagendas da ANPPS contempladas, o levantamento de dados centrou-se nos editais/chamadas lançados pelas FAP, nas atas de julgamento do Comitê Gestor do PPSUS em cada UF e no Portal Pesquisa Saúde do MS, disponível no 
endereço eletrônico http://pesquisasaude. saude.gov.br/.

Na segunda parte, adotou-se o 'Grau de Implantação’ definido por Vieira-Da-Silva \& Formigli (1994) como característica ou atributo para avaliar o grau de implantação do PPSUS nas UF. Segundo Vieira-Da-Silva et al. (2010A), o grau de implantação de uma intervenção divide-se em três estágios, quais sejam: Incipiente $(\geq 0 \quad$ e $<33,3 \%)$; Intermediário ( $\geq 33,3 \%$ e $<66,66 \%)$; e Avançado ( $\geq 66,6 \%$ ).

A estratégia de pesquisa adotada foi o estudo de casos múltiplos (YIN, 2001), baseado na observação e busca ativa nos documentos que retratam a execução do programa nas UF. Os critérios adotados foram: 1) o número de edições do PPSUS; 2) o modelo adotado na definição de prioridade de pesquisa do PPSUS; 3) o sistema de julgamento contido no DDT do PPSUS; e 4) o processo de A\&A dos projetos contratados pelo PPSUS, conforme descrito no quadro 1 . Os critérios 2,3 e 4 fazem parte dos 'componentes' do modelo lógico do PPSUS.

Quadro 1. Matriz de critérios para avaliação do grau de implantação do Programa Pesquisa para o SUS: gestão compartilhada em saúde (PPSUS), nas UF

\begin{tabular}{|c|c|c|c|c|}
\hline \multirow{2}{*}{ Critério } & \multirow{2}{*}{ Ponderação } & \multicolumn{3}{|c|}{ Graus de implantação } \\
\hline & & Não implantado & Parcialmente implantado & Implantado \\
\hline $\begin{array}{l}\text { Número de edições do } \\
\text { PPSUS }\end{array}$ & 30 & $\begin{array}{l}\text { Não participou de nenhuma } \\
\text { edição do PPSUS (0) }\end{array}$ & $\begin{array}{l}\text { Participou de até três edições do } \\
\text { PPSUS (15) }\end{array}$ & $\begin{array}{l}\text { Participou de quatro ou mais edi- } \\
\text { ções do PPSUS ( } 30 \text { ) }\end{array}$ \\
\hline $\begin{array}{l}\text { Modelo adotado na } \\
\text { definição de prioridade } \\
\text { de pesquisa do PPSUS }\end{array}$ & 15 & $\begin{array}{l}\text { Não utilizou o modelo de } \\
\text { definição de prioridades de } \\
\text { pesquisa do PPSUS (0) }\end{array}$ & $\begin{array}{l}\text { Utilizou em até três edições do PP- } \\
\text { SUS o modelo de definição de priori- } \\
\text { dades de pesquisa do PPSUS }(7,5)\end{array}$ & $\begin{array}{l}\text { Utilizou em quatro ou mais edições } \\
\text { o modelo de definição de priorida- } \\
\text { des de pesquisa do PPSUS (15) }\end{array}$ \\
\hline $\begin{array}{l}\text { Sistema de julgamento } \\
\text { contido no DDT do } \\
\text { PPSUS }\end{array}$ & 15 & $\begin{array}{l}\text { Não utilizou o sistema de } \\
\text { julgamento contido no DDT } \\
\text { do PPSUS (0) }\end{array}$ & $\begin{array}{l}\text { Utilizou em até três edições o siste- } \\
\text { ma de julgamento contido no DDT } \\
\text { do PPSUS }(7,5)\end{array}$ & $\begin{array}{l}\text { Utilizou em quatro ou mais edições } \\
\text { o sistema de julgamento contido } \\
\text { no DDT do PPSUS (15) }\end{array}$ \\
\hline $\begin{array}{l}\text { Processo de A\&A dos } \\
\text { projetos contratados } \\
\text { pelo PPSUS }\end{array}$ & 10 & $\begin{array}{l}\text { Não utilizou o processo de } \\
\text { A\&A dos projetos contrata- } \\
\text { dos pelo PPSUS (0) }\end{array}$ & $\begin{array}{l}\text { Utilizou em até três edições o pro- } \\
\text { cesso de A\&A dos projetos contrata- } \\
\text { dos pelo PPSUS (5) }\end{array}$ & $\begin{array}{l}\text { Utilizou em quatro ou mais edições } \\
\text { o processo de A\&A dos projetos } \\
\text { contratados pelo PPSUS (10) }\end{array}$ \\
\hline
\end{tabular}

Fonte: Adaptado de Vieira-da-Silva et al. (2010).

\section{Delimitação do estudo}

Foram incluídas, neste estudo, as edições do PPSUS lançadas no período compreendido entre 2004 e 2012. Foram excluídas as edições 2002, considerada piloto na implantação do programa, e a edição 2015, por encontrar-se em fase inicial de execução (de elaboração dos editais para seleção dos projetos de pesquisa).

\section{Resultados}

No período de 2004 a 2012, foram contratados 2.923 projetos de pesquisa científica, tecnológica e/ou de inovação, resultantes das seleções feitas nas cinco edições lançadas pelo PPSUS, totalizando investimentos no valor global de $\mathrm{R} \$ 274.463 .600,00$, sendo $\mathrm{R} \$$ 161.915.000,00 (59\%) oriundos do MS e R\$ $112.548 .600,00$ (41\%) do Sistema FAP.

O total de recursos investidos, assim como o número de projetos de pesquisa aprovados por UF, são descritos no gráfico 1. Destaca-se que as UF que apresentaram os maiores investimentos no programa foram: Minas Gerais, com R\$ 45,5 milhões (16,6\%) aplicados em 251 projetos (8,6\%); São Paulo, com R \$ 34,2 milhões $(12,5 \%)$ em 204 projetos $(6,7 \%)$; 
e Rio de Janeiro, com R \$ 32,3 milhões (11,8\%) em 169 projetos (5,8\%). As UF de Roraima, de Rondônia e do Amapá foram as que apresentaram os menores investimentos nesse período:
$\mathrm{R} \$ 0,7$ milhões $(0,2 \%)$ aplicados em 12 projetos $(0,4 \%) ; \mathrm{R} \$ 0,8$ milhões $(0,3 \%)$ em 14 projetos (0,5\%); e R $\$ 0,9$ milhões $(0,3 \%)$ em 18 projetos $(0,6 \%)$, respectivamente.

Gráfico 1. Número de projetos aprovados e recursos aportados, por UF, no período de 2002 a 2012

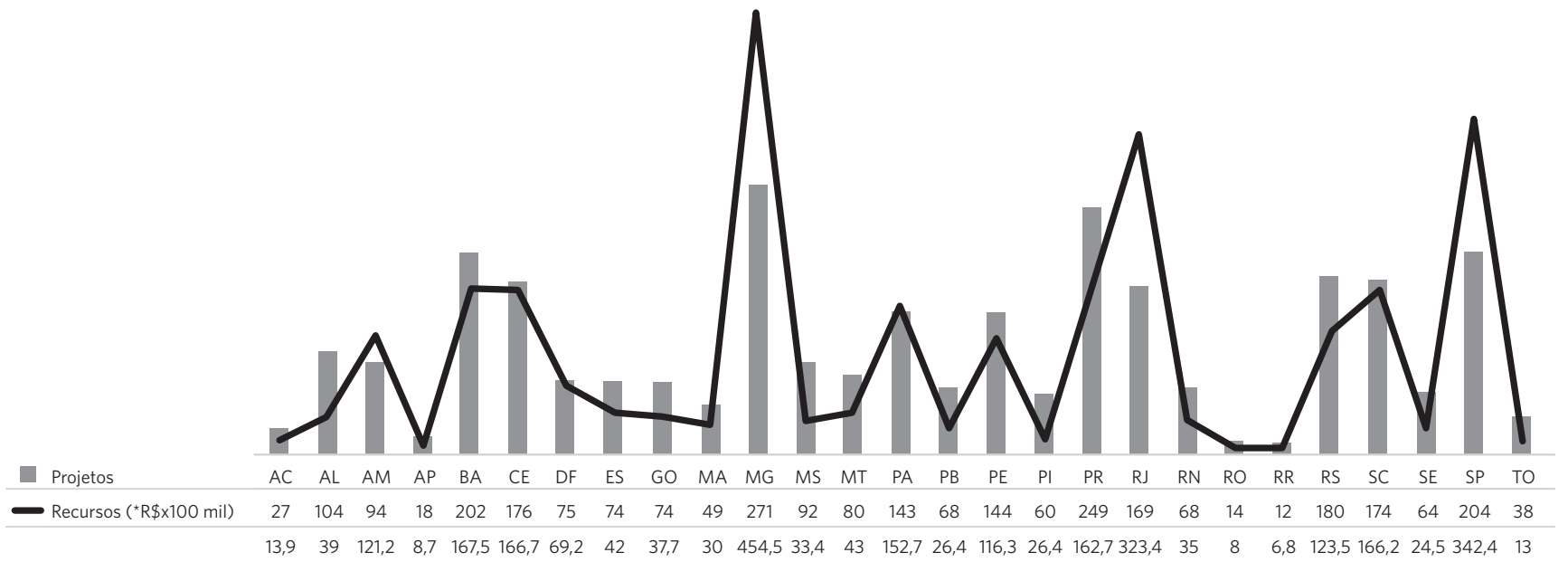

Fonte: BRASIL (2016).

*Valores em R\$ e multiplicados por 100 mil para apresentação no gráfico.

Analisando-se a distribuição de recursos e de projetos por região, constatou-se que a região Sudeste $(42 \%)$ concentra o maior volume de recursos investidos e a região Nordeste, o maior número de projetos aprovados (32\%), conforme indicado no gráfico 2.

Gráfico 2. № de projetos aprovados e recursos financeiros aportados, por região, no período de 2004 a 2012

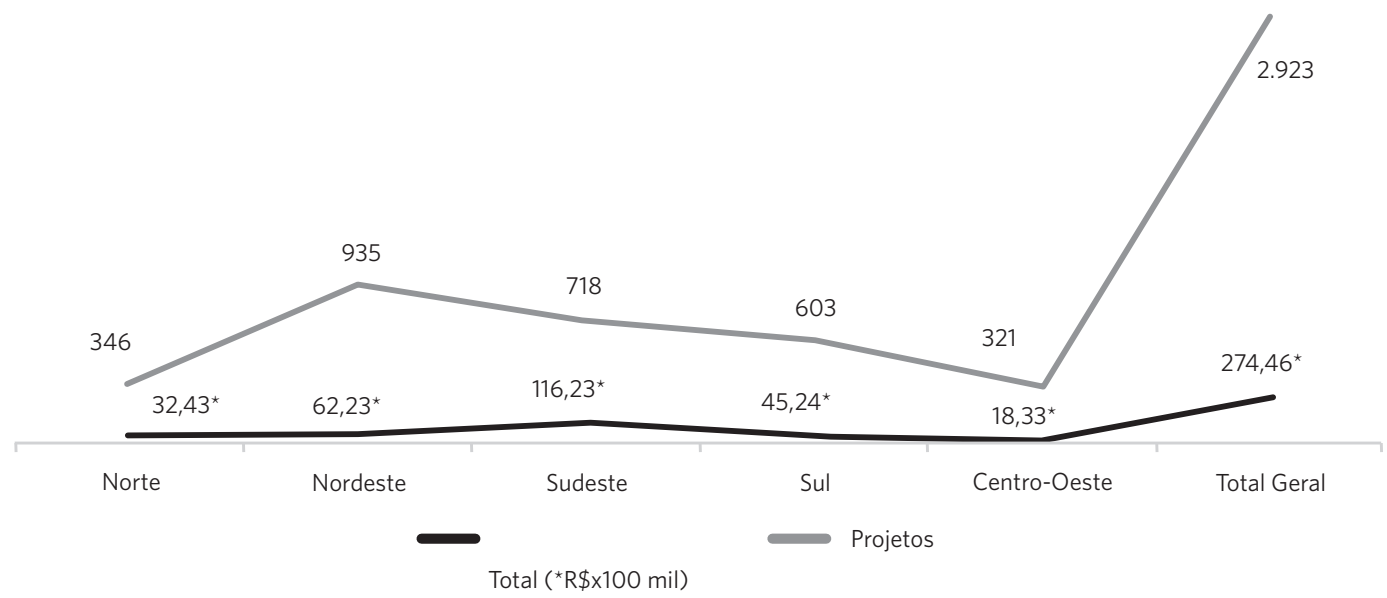

Fonte: BRASIL (2016).

*Valores em R\$ e multiplicados por 100 mil para apresentação no gráfico. 
Ranqueando-se as subagendas da ANPPS com maior número de projetos aprovados, identificou-se uma convergência dos temas pesquisados nas regiões do País, destacando-se: doenças transmissíveis (presentes em todas as regiões); pesquisa clínica
(Sudeste, Sul, Centro-Oeste e Norte); avaliação de tecnologias em saúde e doenças crônicas não transmissíveis (Sudeste, Sul e Nordeste). O gráfico 3 apresenta as cinco subagendas mais pesquisadas no âmbito do PPSUS, segundo as regiões do País.

Gráfico 3. Distribuição das principais subagendas, mais pesquisadas no âmbito do PPSUS, segundo as regiões do País

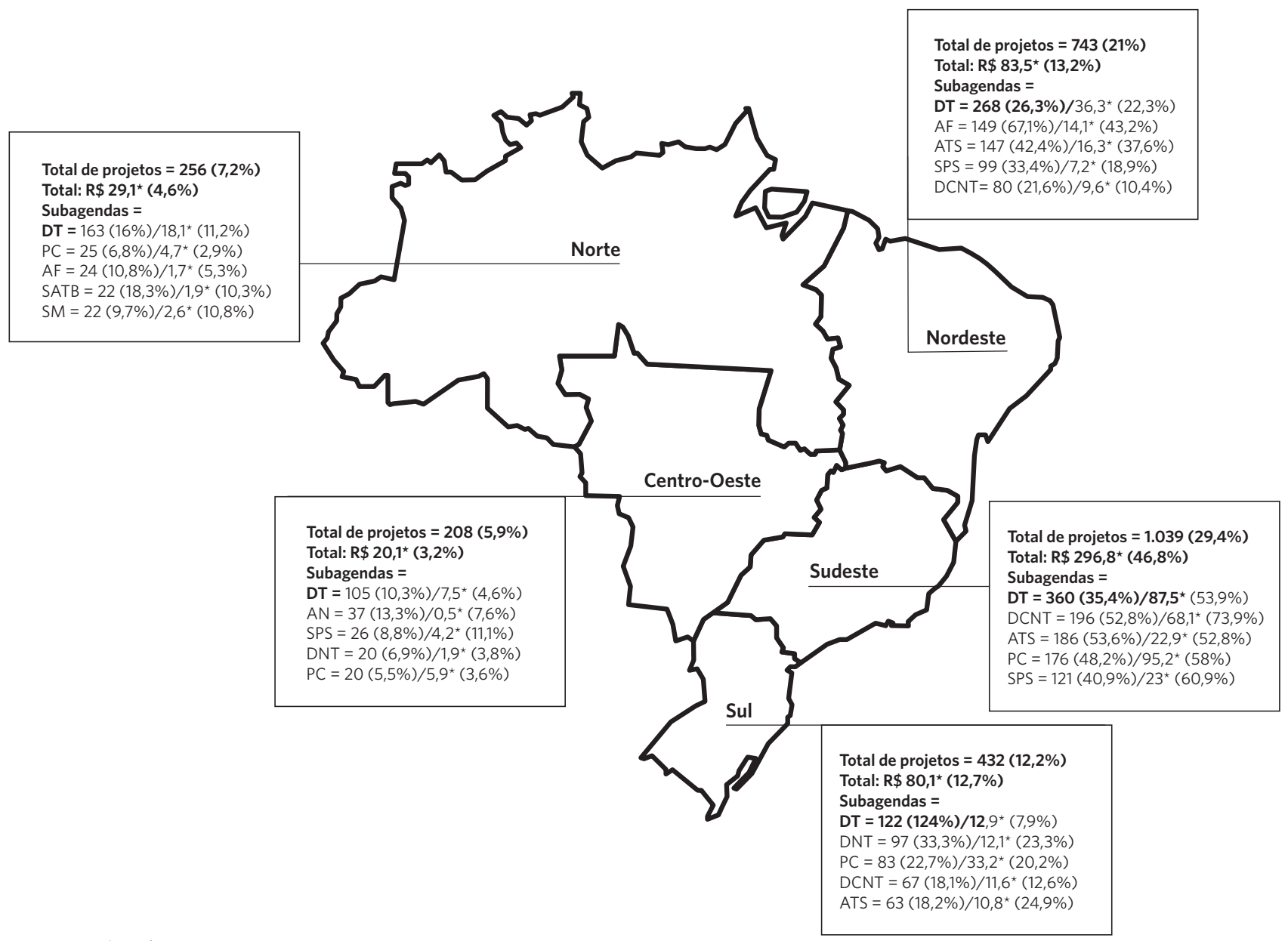

Fonte: BRASIL (2016).

*Valores em R\$ e multiplicados por 100 mil para apresentação no gráfico.

Os resultados obtidos, a partir dos critérios utilizados para avaliação do grau de implantação do PPSUS entre os anos 2004 e 2012, e a respectiva situação de cada UF, encontram-se descritos no quadro 2. Entre as $27 \mathrm{UF}$, verificou-se que todas participaram de, pelo menos, uma das cinco edições do PPSUS lançadas entre 2004 e 2012. 
Quadro 2. Avaliação do grau de implantação do Programa Pesquisa para o SUS: gestão compartilhada em saúde (PPSUS), nas UF

\begin{tabular}{|c|c|c|c|c|c|c|c|c|}
\hline \multirow{3}{*}{ UF } & \multicolumn{2}{|c|}{$\begin{array}{l}\text { Critério 1. Número de edições } \\
\text { do PPSUS }\end{array}$} & \multicolumn{2}{|c|}{$\begin{array}{c}\text { Critério 2: Utilização do Modelo } \\
\text { de Definição de Prioridades de } \\
\text { Pesquisa }\end{array}$} & \multicolumn{2}{|c|}{$\begin{array}{c}\text { Critério 3: Utilização do Sistema } \\
\text { de Julgamento do DDT }\end{array}$} & \multicolumn{2}{|c|}{$\begin{array}{l}\text { Critério 4: Utilização do } \\
\text { Processo de A\&A do DDT }\end{array}$} \\
\hline & Pontuação & Situação & Pontuação & Situação & Pontuação & Situação & Pontuação & Situação \\
\hline & $\begin{array}{l}\text { (0) nenhuma } \\
\text { (15) até } 3 \\
\text { (30) a partir de } 4\end{array}$ & $\begin{array}{l}\text { (1) } \mathrm{NI} \\
\text { (2) } \mathrm{Pl} \\
\text { (3) I }\end{array}$ & $\begin{array}{l}\text { (0) nenhuma } \\
(7,5) \text { até } 3 \\
(15) \text { a partir de } 4\end{array}$ & $\begin{array}{l}\text { (1) } \mathrm{NI} \\
\text { (2) } \mathrm{PI} \\
\text { (3) I }\end{array}$ & $\begin{array}{l}\text { (0) nenhuma } \\
(7,5) \text { até } 3 \\
(15) \text { a partir de } 4\end{array}$ & $\begin{array}{l}\text { (1) } \mathrm{NI} \\
\text { (2) } \mathrm{Pl} \\
\text { (3) I }\end{array}$ & $\begin{array}{l}\text { (0) nenhuma } \\
\text { (5) até } 3 \\
\text { (10) a partir de } 4\end{array}$ & $\begin{array}{l}\text { (1) } \mathrm{NI} \\
(2) \mathrm{PI} \\
\text { (3) I }\end{array}$ \\
\hline$A L$ & 30 & I & 15 & 1 & 15 & I & 10 & I \\
\hline AM & 30 & I & 15 & I & 15 & I & 10 & I \\
\hline BA & 30 & 1 & 15 & I & 15 & I & 10 & I \\
\hline$C E$ & 30 & I & 15 & I & 15 & I & 10 & I \\
\hline ES & 30 & I & 15 & I & 15 & I & 10 & I \\
\hline MA & 30 & I & 15 & I & 15 & I & 10 & I \\
\hline$M G$ & 30 & 1 & 15 & I & 15 & I & 10 & I \\
\hline MS & 30 & I & 15 & I & 15 & I & 10 & 1 \\
\hline MT & 30 & 1 & 15 & 1 & 15 & 1 & 10 & 1 \\
\hline PA & 30 & I & 15 & I & 15 & I & 10 & I \\
\hline PB & 30 & I & 15 & 1 & 15 & I & 10 & I \\
\hline PE & 30 & 1 & 15 & I & 15 & I & 10 & I \\
\hline $\mathrm{Pl}$ & 30 & I & 15 & I & 15 & I & 10 & I \\
\hline$P R$ & 30 & I & 15 & I & 15 & I & 10 & I \\
\hline RJ & 30 & I & 15 & 1 & 15 & I & 10 & I \\
\hline RN & 30 & 1 & 15 & I & 15 & I & 10 & I \\
\hline RS & 30 & I & 15 & I & 15 & I & 10 & 1 \\
\hline SC & 30 & 1 & 15 & 1 & 15 & I & 10 & 1 \\
\hline SE & 30 & 1 & 15 & 1 & 15 & 1 & 10 & 1 \\
\hline SP & 30 & 1 & 15 & 1 & 15 & 1 & 10 & 1 \\
\hline$A C$ & 15 & $\mathrm{PI}$ & 7,5 & $\mathrm{PI}$ & 7,5 & $\mathrm{Pl}$ & 5 & PI \\
\hline AP & 15 & PI & 7,5 & $\mathrm{PI}$ & 7,5 & $\mathrm{Pl}$ & 5 & PI \\
\hline DF & 15 & PI & 7,5 & $\mathrm{PI}$ & 7,5 & $\mathrm{PI}$ & 5 & $\mathrm{PI}$ \\
\hline GO & 15 & PI & 7,5 & PI & 7,5 & PI & 5 & PI \\
\hline $\mathrm{RO}$ & 15 & PI & 7,5 & $\mathrm{PI}$ & 7,5 & PI & 5 & $\mathrm{PI}$ \\
\hline $\mathrm{RR}$ & 15 & PI & 7,5 & PI & 7,5 & $\mathrm{Pl}$ & 5 & PI \\
\hline TO & 15 & PI & 7,5 & PI & 7,5 & PI & 5 & PI \\
\hline
\end{tabular}

Fonte: Elaboração própria.

DDT: Documento de Diretrizes Técnicas do Programa PPSUS

NI: Não Implementado

PI: Parcialmente Implementado

I: Implementado. 
Da análise realizada nas 27 UF, observou-se que o PPSUS obteve a pontuação máxima em 20 UF, sendo considerado como uma intervenção que adotou o preconizado no DDT, tendo sido classificado como 'implantado'. Todas as 16 UF das regiões Sul, Sudeste e Nordeste compõem esse grupo. Entre as 11 UF das regiões Norte e Centro-Oeste, o programa atingiu esse patamar no Amazonas, no Pará, em Mato Grosso do Sul e em Mato Grosso. Nas demais, o PPSUS encontra-se na situação de 'parcialmente implantado'.

\section{Discussão}

A existência do documento de 'Diretrizes Técnicas' (BRASIL, 2014B), do 'Guia PPSUS: Seleção de prioridades de pesquisa em saúde' (BRASIL, 2009), assim como a do modelo lógico do PPSUS (OLIVEIRA, 2008), explicitando a teoria do programa, contribuiu para a implantação e execução do PPSUS no território nacional. As desigualdades regionais e intrarregionais ligadas à infraestrutura das FAP, sua capacidade econômica para aportar contrapartida financeira ao programa, a situação de saúde de cada estado, a massa crítica instalada em universidades e centros de pesquisa, e as prioridades de pesquisa de cada UF foram analisadas como características locais, requerendo soluções diferenciadas, distantes das receitas outrora utilizadas. Essa postura dos partícipes federais (MS e CNPq) do PPSUS transformou dificuldades em oportunidades e contribuiu para a implantação do programa em âmbito nacional.

Preliminarmente à discussão acerca dos resultados alcançados com a execução do PPSUS, será enfocado o grau de implantação do programa nas UF. Antes de serem formatados os critérios de avaliação, adotou-se as recomendações descritas por Denis \& Champagne (1997) referentes à teoria do programa, à identificação das práticas necessárias à implantação da intervenção, à descrição das práticas correntes dos partícipes e à análise da variação na implantação, a partir da variação das características contextuais.

A participação das UF nas cinco edições do programa ocorreu de forma não linear. As ausências em determinadas edições foram motivadas por diversos fatores, entre os quais a capacidade financeira da FAP, a inexistência de FAP na UF e, quando presentes, problemas referentes à regularidade fiscal, que impediram a celebração de convênios com o CNPq. Além disso, na edição 2011, somente dez UF participaram do programa. Esse grupo foi selecionado pelo próprio MS, considerando as peculiaridades daquela edição, que deveria englobar, também, a 'implantação das redes de pesquisa' nos estados (BRASIL, 2014A).

Portanto, na avaliação do 'grau de implantação' do PPSUS nas UF do País, adotou-se, entre outros critérios, o número de participação nas cinco edições lançadas. Constatou-se que, em 20 das 27 UF, o PPSUS foi avaliado como sendo um programa 'implantado'. Isto significa que aquelas UF participaram de quatro edições e seguiram o que preconiza o documento DDT do PPSUS.

Nas demais UF, com predomínio da região Norte, o programa é avaliado como 'parcialmente implementado'. Isto não significa que não foram adotados os ritos previstos no documento, mas constatou-se que as participações daquelas UF ficaram aquém das quatro edições definidas como parâmetros de avaliação e enquadramento na situação de 'implantado'.

Independentemente do grau de implantação, os resultados encontrados, com a execução do programa, demonstram a magnitude alcançada pelo PPSUS em relação aos investimentos globais (aumento de 325,8\% entre a edição 2004 e a 2012), ao número de projetos submetidos (7.093), ao número de aprovados (2.923), ao aporte de recursos em todas as UF do País - inclusive, com contrapartida financeira de todas as FAP -, assim como em relação ao avanço na gestão compartilhada 
das atividades, a observância à descentralização efetiva do poder decisório às FAP nas atividades sob sua responsabilidade.

Outro aspecto não menos importante foi a autonomia dada ao CNPq, pelo MS, com relação à gestão dos recursos federais aplicados no PPSUS e à celebração de convênios que possibilitaram a transferência efetiva, no tempo preciso, de recursos às FAP, garantindo a execução do programa em cada UF.

No âmbito do programa, a questão do poder decisório baseou-se em uma relação amistosa entre os partícipes, sem hierarquizações, mas centrado na responsabilidade e na experiência de cada um, na execução da atividade. Segundo Foucault (1982), só existirá realmente a descentralização do poder decisório entre os partícipes de uma intervenção quando não houver uma relação de hierarquia entre eles. De fato, além dos recursos, a gestão do PPSUS adota o modelo de descentralização das decisões baseado na etapa do programa.

O crescimento do programa, ao longo dos anos, fica evidenciado pela adesão das UF, das SES, e pelo interesse demonstrado pela comunidade científica, de participar das etapas, com destaque para as oficinas de definição das prioridades de pesquisa, no quantitativo de projetos submetidos em resposta às chamadas lançadas.

Identificou-se ampla participação de pesquisadores, gestores, técnicos das SES e representantes das FAP nos seminários parciais e finais de A\&A. Este esforço conjunto assegura que os recursos investidos nas ações de fomento à pesquisa científica, tecnológica e de inovação contribuem para a resolução dos problemas prioritários de saúde demandados em cada UF do País, além de reforçar a necessidade de promover a descentralização e a desconcentração dos investimentos federais no setor saúde.

Dada a histórica desigualdade regional e intrarregional no fomento à pesquisa em saúde, o PPSUS constitui-se não só em um fator de desenvolvimento local, mas também em um orientador de ações estratégicas nos serviços de saúde, possibilitando, inclusive, a inserção da pesquisa científica, tecnológica e de inovação em saúde nas agendas das SES.

A definição das prioridades de pesquisa por cada UF é um fator preponderante e também inovador desse processo, e contou, ao longo das edições do programa, com a participação de técnicos das SES, das FAP, do MS, do CNPq, de pesquisadores de instituições de ensino e pesquisa, além de representantes do controle social (BRASIL, 2009). Este último, exercido por intermédio das Conferências de Saúde e dos Conselhos de Saúde.

Nesta etapa, o programa apoia-se firmemente no conhecimento dos gestores do SUS e na expertise dos pesquisadores para identificação e resolução dos problemas de saúde enfrentados em cada UF. Coube às SES, apoiadas pelas FAP, o papel de articular e sistematizar as prioridades de pesquisa que compõem os editais e/ou chamadas lançados no âmbito do PPSUS, nas UF.

O estabelecimento de prioridades de pesquisa orienta a aplicação dos recursos de maneira mais racional, mais focado nas necessidades de saúde, e não apenas para manter linhas de pesquisa nas universidades e/ou centros de pesquisa. Este redirecionamento do fomento pode diminuir os efeitos previstos no desequilíbrio conhecido como 'gap 10/90', onde apenas 10\% dos recursos disponíveis para pesquisa são direcionados às doenças e agravos, que afetam $90 \%$ da população (GLOBAL FORUM FOR HEALTH RESEARCH, 2000).

$\mathrm{O}$ enquadramento dos 2.923 projetos financiados pelo PPSUS no âmbito das subagendas de pesquisa identificou uma convergência de temas por regiões do País, o que caracteriza um perfil nacional de prioridades de pesquisa no setor saúde, mesmo sendo respeitadas as vocações regionais e os problemas locais de saúde. A subagenda 'doenças transmissíveis' - que foi definida localmente, mas está presentes nas cinco regiões do País - conta com 1.018 (34\%) projetos financiados. 
Em um ambiente com escassez de recursos aliada à emergência e à reemergência de doenças, é fundamental aproximar o universo científico da atividade de atenção à saúde e possibilitar que a saúde pública interaja, de forma mais efetiva, com a CTIS. Esse estreitamento entre produtores e usuários do conhecimento pode contribuir com a geração, e consequente incorporação, pelo SUS, de soluções pelas quais a sociedade brasileira tanto anseia.

A avaliação como campo conceitual e de trabalho não está bem definida: o papel do avaliador carece de clareza e os conceitos da área são utilizados de formas diferentes. Além disso, frequentemente são criados novos conceitos, que redundam em uma imensa diversidade terminológica (VIEIRADA-SILVA; FORMIGLI, 1994), indicando a riqueza do campo da avaliação e, ao mesmo tempo, gerando dificuldades para uma comunicação clara sobre o tema (PATTON, 1982). Isso pôde ser comprovado durante os seminários de A\&A dos projetos aprovados, quando o PPSUS conseguiu compor uma mesa de discussão com membros das FAP, da comunidade científica e dos serviços do SUS, criando novos conceitos e gerando novas incorporações dos resultados alcançados.

Além dos resultados científicos, evidenciados pelas publicações de artigos, livros e patentes, destaca-se, também, a formação de recursos humanos para o setor saúde, considerando que teses e dissertações foram desenvolvidas e concluídas com o apoio do programa. Esta vertente de apoio contribui para a qualificação de recursos humanos, com a perspectiva de incorporação dos conhecimentos e das tecnologias produzidas às ações e aos serviços do SUS, gerando mudanças de pensamento, de comportamento e programáticas ou organizacionais, nas práticas e na cultura, como resultado da aprendizagem, durante o processo avaliativo. Acredita-se que adotar o pressuposto de foco na utilidade requer a necessária coerência entre os propósitos e procedimentos da avaliação (FIGUEIRÓ ET AL. 2012).
Há avanços na formação de mestres e doutores no País. No entanto, as regiões Norte e Centro-Oeste detêm pouco mais de $15 \%$ dessa massa crítica especializada. Estudo do Centro de Gestão e Estudos Estratégicos (CGEE, 2010) indica que 70\% dos doutores do País são formados na região Sudeste, fato que reforça a endogenia da formação e dificulta a fixação desses profissionais em outras regiões brasileiras.

Além das publicações técnicas e científicas sob responsabilidade dos pesquisadores apoiados, o Decit/SCTIE-MS formatou o 'Encontro Nacional do PPSUS: iniciativas inovadoras de pesquisa em saúde' (BRASIL, 2014C), realizado em Brasília, com o objetivo de ampliar a divulgação dos resultados mais promissores alcançados pelos projetos apoiados entre os membros da academia e gestores do SUS.

No âmbito do PPSUS, a avaliação dos resultados alcançados e sua divulgação estão bem estruturadas. No entanto, é preciso formatar instrumentos que facilitem ou permitam que o conhecimento gerado e as intervenções sanitárias resultantes sejam efetivamente incorporados às políticas $\mathrm{e}$ ações de saúde pública (MOREL, 2004). Segundo Toma et al. (2011), é preciso fortalecer as relações entre pesquisadores e gestores do SUS, pois suas atuações nas dimensões do 'fazer ciência' e ‘tomar decisões' não os aproxima, e pode dificultar e/ou impossibilitar possíveis incorporações, no SUS, das soluções produzidas pelas pesquisas.

Na quinta edição do DDT do PPSUS (BRASIL, 2014B, P. 17), ficou estabelecido que caberá às SES, a partir dos resultados apresentados nos seminários finais de A\&A, a "análise do potencial e incentivo à incorporação dos resultados das pesquisas nos serviços de saúde", inclusive a obrigação de "estabelecer estratégias" que possibilitem essa incorporação. As estratégias utilizadas no PPSUS, para disseminação das informações em saúde, buscam envolver todos os atores e setores dos mais diversos níveis, possibilitando a 
aproximação da academia dos serviços de saúde, a participação efetiva dos gestores de saúde no programa e o envolvimento do Conselho Nacional de Secretários de Saúde (Conass) e do Conselho Nacional de Secretarias Municipais de Saúde (Conasems) nas ações estratégicas do programa.

\section{Considerações finais}

A execução do PPSUS possibilitou a aproximação entre os sistemas estaduais de saúde e de ciência e tecnologia, contribuiu para a consolidação do sistema estadual de ciência e tecnologia, incentivou a formação de recursos humanos (mestres e doutores) na área da saúde, atendeu às necessidades locais de pesquisa em saúde e contribuiu para a desconcentração dos investimentos feitos pelo governo federal no setor saúde. Estas contribuições demonstram que o programa obteve êxito, e que a formação de seu modelo lógico e a utilização do DDT foram instrumentos essenciais para a sua implantação nas UF do País.

\section{Agradecimentos}

Este trabalho não teria sido desenvolvido sem a solidária colaboração e o empenho de Luci Sheffer e Celia Aly, do Departamento de Monitoramento e Avaliação do SUS, do Ministério da Saúde; de Daniela Scarabucci, da Coordenação de Biociências, do Conselho Nacional de Desenvolvimento Científico e Tecnológico; e de Sidney Domingos, do Hospital Alemão Oswaldo Cruz, de São Paulo.

\section{Colaboradores}

Todos os autores contribuiram substancialemnte na concepção, no planejamento, na análise e na interpretação dos dados.

\section{Referências}

BARROS, F. A. F. Os desequilíbrios regionais da produção técnico-científica. São Paulo Perspec., São Paulo, v. 14, n. 3, p. 12-19, jul./set. 2000

BRASIL. Ministério da Saúde. Secretaria de Atenção à Saúde. Implantação das Redes de Atenção à Saúde e outras estratégias da SAS. Brasília, DF: Ministério da Saúde, 2014a.

Ministério da Saúde. Secretaria de Ciência,

Tecnologia e Insumos Estratégicos. Departamento de Ciência e Tecnologia. Descentralização no contexto do fomento à pesquisa em saúde. Rev. Saúde Pública, São
Paulo, v. 45, n. 3, p. 626-30, 2011 .

Ministério da Saúde. Secretaria de Ciência, Tecnologia e Insumos Estratégicos. Departamento de Ciência e Tecnologia. Seleção de prioridades de pesquisa em saúde: guia PPSUS. Brasília, DF: Ministério da Saúde, 2009 (Série A. Normas e Manuais Técnicos).

Ministério da Saúde. Secretaria de Ciência, Tecnologia e Insumos Estratégicos. Departamento de Ciência e Tecnologia. Pesquisas estratégicas para o sistema de saúde: PESS. Brasília, DF: Ministério da Saúde, $2011 b$ (Série B. Textos Básicos de Saúde). 
Ministério da Saúde. Secretaria de Ciência, Tecnologia e Insumos Estratégicos. Departamento de Ciência e Tecnologia. Programa de Pesquisa para o SUS: gestão compartilhada em saúde: diretrizes técnicas. 5. ed. Brasília, DF: Ministério da Saúde, 2014b. Disponível em: <http://u.saude.gov.br/images/pdf/2015/marco/30/diretrizes-tecnicas-PPSUS-2014.pdf $>$. Acesso em: 2 fev. 2017.

Ministério da Saúde. Secretaria de Ciência, Tecnologia e Insumos Estratégicos. Departamento de Ciência e Tecnologia. Encontro Nacional do PPSUS: iniciativas inovadoras de pesquisa em saúde. Brasília, DF: Ministério da Saúde, 2014c. Disponível em: <http:// bvsms.saude.gov.br/bvs/publicacoes/encontro_nacional_ppsus.pdf $>$. Acesso em: 2 fev. 2017.

Ministério da Saúde. Secretaria de Ciência, Tecnologia e Insumos Estratégicos. Departamento de Ciência e Tecnologia. Sistema Pesquisa Saúde. Disponível em: $<$ http://www.saude.gov.br/pesquisasaude>. Acesso em: 3 fev. 2016.

\section{CENTRO DE GESTÃO E ESTUDOS ESTRATÉGICOS} (CGEE). Descentralização do fomento à ciência, tecnologia e inovação no Brasil. Brasília, DF: CGEE, 2010. Disponível em: <http://www.cgee.org.br/publicacoes/ descentralizacao.php>. Acesso em: 15 nov. 2016.

CHEN, H. T. Theory-Driven Evaluations. London: Sage Publication, 1990.

DENIS, J. L.; CHAMPAGNE, F. Análise da implantação. In: HARTZ, Z. M. A. (Org.). Avaliação em Saúde: dos modelos conceituais à prática na análise da implantação de programas. Rio de Janeiro: Fiocruz, 1997, p. 49-88.

FOUCAULT, M. Microfísica do poder. Rio de Janeiro: Graal, 1982.

FIGUEIRÓ, A. C. et al. Usos e influência da avaliação em saúde em dois estudos sobre o Programa Nacional de Controle da Dengue. Cad. Saúde Pública, Rio de Janeiro, v. 28, n. 11, p. 2095-2105, nov. 2012.

GLOBAL FORUM FOR HEALTH RESEARCH. The 10/90 Report on Health Research. Geneva: WHO, 2000.
MOREL, C. M. A pesquisa em saúde e os objetivos do milênio: desafios e oportunidades globais, Soluções e políticas nacionais. Ciência \&t Saúde Coletiva, Rio de Janeiro, v. 9, n. 2, p. 261-270, 2004.

OLIVEIRA, M. M. Programa Pesquisa para o SUS: gestão compartilhada em Saúde - PPSUS: construção de modelo lógico e da matriz de medidas avaliativas. 2008. 132 f. Dissertação (Mestrado em Ciências da Saúde) - Programa de Pós-Graduação em Ciências da Saúde, Faculdade de Ciências da Saúde, Universidade de Brasília, Brasília, 2008.

PATTON, M. Q. Practical evaluation. Beverly Hills: Sage Publications, 1982.

TOMA, T. S. et al. PPSUS e o desafio da incorporação dos resultados das pesquisas no Estado de São Paulo. Bis, São Paulo, v. 13, n. 1, p. 15-25, abr. 2011.

VIEIRA-DA-SILVA, L. M. Avaliação de políticas e programas de saúde. Rio de Janeiro: Fiocruz, 2014.

VIEIRA-DA-SILVA, L. M. Conceitos, abordagens e estratégias para a avaliação em saúde. In: HARTZ, Z. M. A.; SILVA, L. M. V. (Org.). Avaliação em saúde: dos modelos teóricos à prática na avaliação de programas e sistemas de saúde. Rio de Janeiro; Salvador: Fiocruz; Edufba, 2005, p. 15-40.

VIEIRA-DA-SILVA, L. M.; FORMIGLI, V. L. A. Avaliação em saúde: limites e perspectivas. Cad. Saúde Pública, Rio de Janerio, n. 10, p. 80-91, mar. 1994.

VIEIRA-DA-SILVA, L. M. et al. Avaliação da implantação de programa voltado para melhoria da acessibilidade e humanização do acolhimento aos usuários na rede básica. Salvador, 2005-2008. Rev. Bras. Saúde Mater. Infant., Recife, n. 10, supl. n. 1, p. s131-s143, nov. 2010.

YIN, R. K. Estudo de caso: planejamento e métodos. 2. ed. Porto Alegre: Bookman, 2001.

Recebido para publicação em abril de 2016

Versão final em outubro de 2016

Conflito de interesses: inexistente

Suporte financeiro: não houve 